EOmmun:HEITh, Communication et organisation

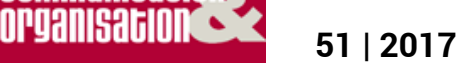

Les nouvelles cultures de l'information dans les organisations

\title{
La mort, l'humour et les stratégies de communication numérique
}

Death, humour and digital communication strategies

Tamires Ferreira Coêlho et Marcelo Rodrigo da Silva

\section{OpenEdition}

Journals

Édition électronique

URL : http://journals.openedition.org/communicationorganisation/5541

DOI : 10.4000/communicationorganisation.5541

ISBN : 979-10-300-0146-4

ISSN : $1775-3546$

Éditeur

Presses universitaires de Bordeaux

Édition imprimée

Date de publication : 1 juin 2017

Pagination : 77-90

ISBN : 979-10-300-0162-4

ISSN : $1168-5549$

Référence électronique

Tamires Ferreira Coêlho et Marcelo Rodrigo da Silva, « La mort, l'humour et les stratégies de communication numérique », Communication et organisation [En ligne], 51 | 2017, mis en ligne le 01 juin 2020, consulté le 02 janvier 2021. URL : http://journals.openedition.org/communicationorganisation/ 5541 ; DOI : https://doi.org/10.4000/communicationorganisation.5541 


\title{
La mort, l'humour et les stratégies de communication numérique
}

\author{
Tamires ferreira Coêlho' ${ }^{1}$, Marcelo Rodrigo da Silva ${ }^{2}$
}

\section{Introduction}

Créer et employer des stratégies de communication efficaces dans la sphère numérique est une nécessité et un besoin, spécialement dans le contexte organisationnel, à l'affût des alternatives et expériences possibles. Le recours à l'humour, l'exploration des matérialités et multisensorialités (Pereira 2012) est un moyen efficace pour attirer l'attention du public - toujours plus sollicitée au regard de la multiplicité des stimulations et contenus.

Dans ce texte, notre approche de l'humour se fera sous l'angle de la construction des stratégies de communication, et plus particulièrement pour traiter un thème ardu, la mort, à partir d'un modèle d'organisation classiquement peu étudié : un cimetière. Nous analysons les tactiques d'annonces véhiculées sur la page Facebook du cimetière " Jardim da Ressureição - Cemitério Parque » (https://www.facebook.com/cemijardimdaressureicao/), situé à Teresina/PI qui a réussi la prouesse de développer un outil institutionnel, non seulement populaire, et dont la page est celle qui compte le plus de suiveurs dans cette catégorie au Brésil ${ }^{3}$ sur Facebook, résultat obtenu après la restructuration de la page deux ans après son lancement (Gomes et al. 2016).

Pour observer et problématiser les caractéristiques récurrentes des créations et approches de cette page de Facebook, nous avons sélectionné quatre annonces ${ }^{4}$ de 2016 qui font référence à des campagnes de grande mobilisation

\footnotetext{
1 Professeure du Département de Sciences Sociales, Journalisme et Service Social à l'Université Fédérale d'Ouro Preto (UFOP-Mariana), Doctorante à l'Université Fédérale de Minas Gerais (UFMG-Belo Horizonte) et à l'École des Hautes Études en Sciences de l'Information et de la Communication (CELSA-Paris) (avec une bourse de l'agence CAPES-Brésil), Membre du Laboratoire GRIPIC (CELSA-Paris) et des Groupes de Recherche «Processocom» (UNISINOS-São Leopoldo), «GRIS» (UFMG-Belo Horizonte), «Margem» (UFMG-Belo Horizonte) et du réseau «Red AmLat»; tamiresfcoelho@gmail.com

2 Professeur à l'Université Fédérale de la Paraíba (UFPB-João Pessoa), Doctorant à l'Université Fédérale du Rio Grande do Norte (UFRN-Natal), Membre du Groupe de Recherche «PRAGMA» (UFRN-Natal) et du réseau «Red AmLat»; prof.marcelorodrigo@gmail.com

3 La page compte plus de 71700 fans (donnée relevée le 18 avril 2017).

4 À côté de chaque image, au sein de ce texte, nous avons mis à disposition un code QR pour faciliter la
} 
sociale et d'intérêt public. Ces publications ont été choisies car elles ont fait l'objet du plus grand nombre de j'aime et de réactions ${ }^{5}$ sur la « ligne de temps » : la publication sur la Gay Pride (28 juin) qui a totalisé 1400 j'aime et réactions, 231 partages et 61 commentaires ; celle de la Campagne pour les dons d'organe (23 août) qui a obtenu 1200 j'aime et réactions, 119 partages et 110 commentaires; celle de la Journée contre le Tabac (29 août) qui a atteint 5300 j'aime et réactions, 5700 partages et 264 commentaires ; et celle de la Journée Mondiale de Prévention du Suicide (10 septembre) qui a comptabilisé 2400 j'aime, 184 partages et 65 commentaires.

L'analyse a été faite à la lumière des théories de la communication organisationnelle et du processus de médiatisation et plus spécifiquement des stratégies narratives et discursives mobilisées pour la construction de l'image institutionnelle du cimetière par le biais du réseau social. Nous avons tenu compte de la composition des éléments oraux et visuels dans l'atmosphère sémantique du discours médiatique de l'organisation, à partir de la compréhension de l'articulation du processus de textualisation à " une action individuelle et collective qui produit des "dits" et des "inédits" " (d'Almeida $2015: 105)$.

Le contenu humoristique diffusé par les pages organisationnelles établit des liens entre les pratiques numériques et la "culture de l'information" (Liquète 2014) qui est présente dans ces institutions. Le processus de médiatisation et la « nouvelle architecture communicationnelle médiatique " (Fausto Neto 2010) qui émerge en son sein mettent en lumière de nouveaux protocoles et formes de circulation des sens qui, à leur tour, ne se restreignent pas aux savoirs produits formellement dans le domaine de l'institution. La production de contenus pour internet est imprégnée de savoirs non traditionnels. La constitution de références qui fleurissent sur les réseaux sociaux est, bien plus que pour les autres moyens de communication, basée sur le langage oral, sur les écritures et les réécritures, sur l'association entre le partage et la performance qui déplace les processus communicatifs au-delà des pôles rigides de production et de réception.

Il est juste de souligner que le développement de cette proposition tient compte du contexte spécifique de la capitale de l'état du Piauí en association avec le public mobilisé par la page du cimetière sur Facebook. Teresina est l'une des capitales qui présente le taux le plus élevé de suicides chez les jeunes et les actions institutionnelles du Cemi (comme l'appellent « avec tendresse » les fans et suiveurs) sur les réseaux sociaux sont essentiellement tournées vers

visualisation du document sur internet.

5 Outre l'option « j’aime ", Facebook permet également aux internautes de choisir entre cinq réactions pour chaque publication : «j’adore » (émoticone du cœur), « haha » (émoticone du rire), « wouah » (émoticone de la surprise), « tristesse » (émoticone avec une larme) et " grr » (émoticone de la colère). Pour comptabiliser les " j’aime », Facebook ne tient pas compte de ces réactions. Les chiffres ici présentés ont été contrôlés jusqu’à l'élaboration de cet article en octobre 2016. 
un public jeune ${ }^{6}$ si l'on tient compte du langage élaboré dans les publications et les campagnes ainsi que les sens mobilisés.

Nous soulignons les articulations entre humour et capital social qui renforcent les liens de mise en relation (Siqueira Filho 2011: 10). Diverses valeurs organisationnelles sont diffusées par le biais de l'humour et des stratégies discursives y sont développées (Carrieri 2004) en lien avec le capital immatériel de l'entreprise et qui se rattachent à ses valeurs, à ses flux d'informations, à sa culture et à la façon dont elle se met en relation avec ses publics (Guyot 2012) puisqu'il s'agit d'un instrument de communication puissant qui facilite la transmission d'émotions (Irigaray et al. 2010).

\section{La relation entre la mort et l'humour au sein de la communication du Cemi}

Nous avons initialement fait mention que ce texte part d'une perspective critique de l'organisation, orientée vers la production de signification pour mettre en relief l'aspect relationnel de l'articulation entre l'organisation et l'humanisation (Mumby 2010 ; Siqueira Filho 2011). Nous savons que « [...] les organisations sont des espaces de production et de commercialisation de biens et de services mais qu'elles sont aussi des endroits où l'on produit des normes, valeurs, identités, discours, sociabilités, représentations, conflits, etc. » (d'Almeida 2015 : 18). Néanmoins, face à des publics plus exigeants, il est plus complexe de procéder à la construction d'une scène visible, au partage qui met en lumière certains éléments et en élimine d'autres (d'Almeida 2015). L'appropriation stratégique de l'humour n'y échappe pas.

En tenant compte du processus de médiatisation (Braga 2012) présent dans les pratiques organisationnelles et leur appareillage communicationnel, il n'est donc pas suffisant de créer des profils au sein des réseaux sociaux ou d'incorporer des dispositifs technologiques au quotidien. Tout comme l'on ne peut se contenter d'utiliser l'humour sans discrimination : il est nécessaire de penser aux demandes et aux relations à construire avec les publics, aux expectatives et aux symboles qui circulent déjà socialement, comme nous le verrons par la suite dans les analyses des annonces sélectionnées.

6 « Dans une analyse faite par l'équipe, il a été constaté que les villes qui présentent le plus de suiveurs sont celles de São Paulo (3.976), Rio de Janeiro (2.467) et Teresina (1.791) » selon Gomes et al (2016 : 9-10) bien que le cimetière soit localisé dans la capitale du Piauí. 

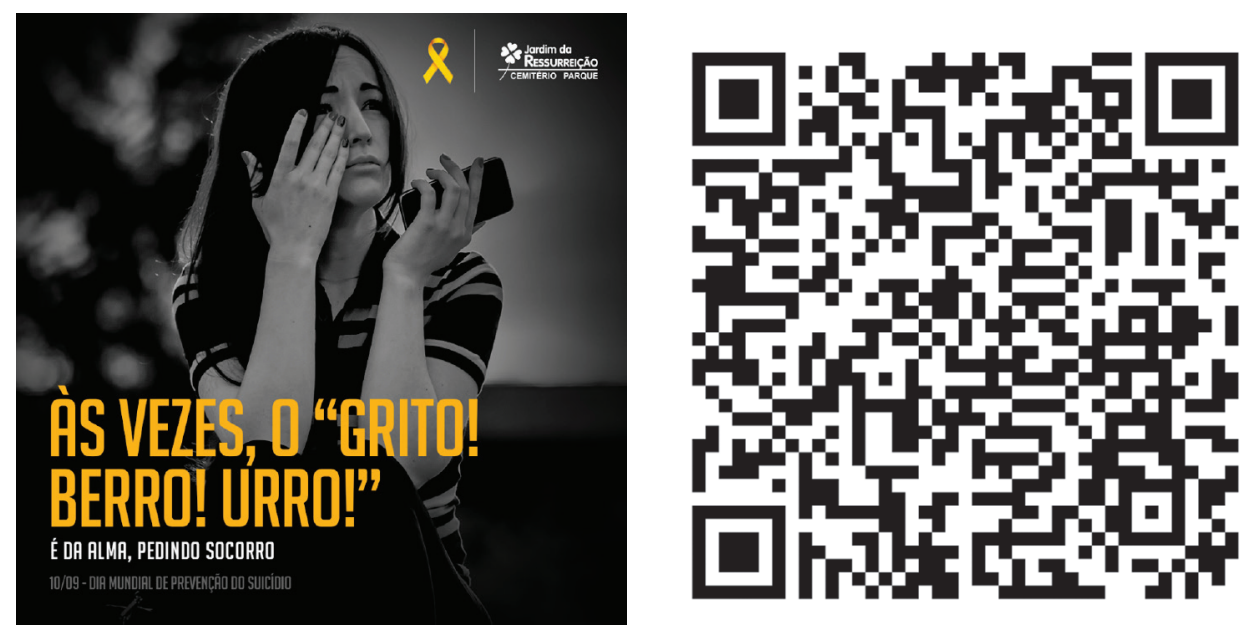

Annonce 1: Journée Mondiale de Prévention contre le suicide.

Source : Page Facebook du Cimetière du Jardin de la Résurrection

La publication fait apparaitre le texte suivant: « Parfois, le "Cri ! Hurlement! Gémissement !"vient de l'âme, un appel au secours. 10/09 - Journée Mondiale de Prévention contre le Suicide [logo du cimetière] ». Immédiatement, les expressions « Cri ! », «Hurlement ! » et « Gémissement ! » établissement une connexion intertextuelle (Maingueneau 1997) qui renvoie à l'univers des sens en lien avec les artistes « pop » contemporains et leurs fans, spécifiquement des artistes qui devinrent célèbres de par leurs productions dans le monde numérique. Dans ce contexte, ces expressions sont couramment utilisées pour ovationner, exalter et glorifier. Ce sont des termes employés comme des " mots d'ordre " ou des " cris de guerre ", dont le but est d'attirer l'attention de manière euphorique pour mettre en évidence et pour soutenir par le biais d'un bruit ou une forte manifestation sonore quelque chose qui est considéré impressionnant ou choquant.

On peut noter que la composition de la publication utilise le sens contextuel qui est couramment employé pour les expressions dans la sphère numérique afin de propulser la publication en faisant usage de la " monnaie sociale » (Berger 2014) qui confère une valeur symbolique à la communication organisationnelle par le biais de l'atmosphère sémantique construite.

De la même façon que les gens utilisent l'argent pour acheter des produits ou des services, ils emploient la monnaie sociale pour obtenir des impressions positives désirées auprès de leur famille, amis et collègues. Donc, pour faire parler les personnes, les entreprises et les organisations doivent faire frapper leur monnaie sociale. Offrir quelque chose pour que les personnes puissent faire bonne impression lorsqu'elles diffusent leurs produits et leurs idées (Berger $2014: 43$ ). 
Cette discussion est également alimentée par le concept de capital social (Siqueira Filho 2011) qui implique une articulation avec l'humour pour renforcer les relations dans la culture organisationnelle puisqu'en « améliorant la confiance, on facilite les changements et l'on encourage la pluralité des visions » (Siqueira Filho $2011: 10$ ).

En pensant que, comme nous l'avons dit préalablement, que l'humour est capable de faire en sorte que les individus soient enclins à construire de fortes relations, en d'autres termes, une confiance mutuelle et un travail collectif, l'humour est donc aussi capable d'activer des réseaux de relations pour viser le bien commun. On peut noter que l'humour peut créer et même être le produit de liens entre les individus. C'est-à-dire contribuer à créer du capital social (Siqueira Filho $2011: 6$ ).

Cette publication enrichit son discours avec des expressions de l'univers symbolique collectif des internautes. Néanmoins, de par la construction de l'énoncé, la rupture de l'attente du lien établi avec un thème sérieux, comme celui de la prévention des suicides, introduit le facteur de surprise qui crée une plus grande implication et stimule l'intérêt de la part du public. La publication conduit le lecteur à faire une pause et à réfléchir aux situations où la demande d'attention, traduite en signaux « hurlants » et perceptibles, peut être le réflexe d'un manque ou de traumatismes affectifs, d'instabilité et débilité émotionnelles, ou même d'états dépressifs couramment associés aux causes de suicide.

L'image utilisée dans la publication renforce le lien du message avec l'un de ses publics les plus importants : les jeunes de Teresina. Teresina est l'une des capitales brésiliennes avec le taux de suicide le plus important chez les jeunes. Ce n'est pas le fait du hasard que l'image représente une jeune femme, qui a l'air triste, qui pleure et qui tient un téléphone, ce qui traduit une relation ironique et indicielle qui s'adresse directement aux jeunes qui utilisent les médias numériques.

Lénoncé joue également avec les sens en lien avec les phénomènes paranormaux lorsqu'il est dit que le « Cri ! Hurlement! Gémissement » est de "l'âme ». Cette âme peut être ou l'essence affective et sensible d'un être humain en souffrance et qui "appelle au secours " ou peut être interprété comme une manifestation paranormale d'une créature non matérialisée dans son corps, ce qui renforce les sens qui gravitent autour de la mort et de la propre configuration symbolique de l'espace du cimetière.

Les couleurs sont également des éléments importants : la composition en noir et blanc qui suggère la tristesse, "l'absence de couleur ", est en contraste avec le jaune qui est la caractéristique de la campagne "Septembre Jaune » qui aborde le thème de la prévention du suicide et qui recueille une adhésion massive des institutions et des entreprises. 

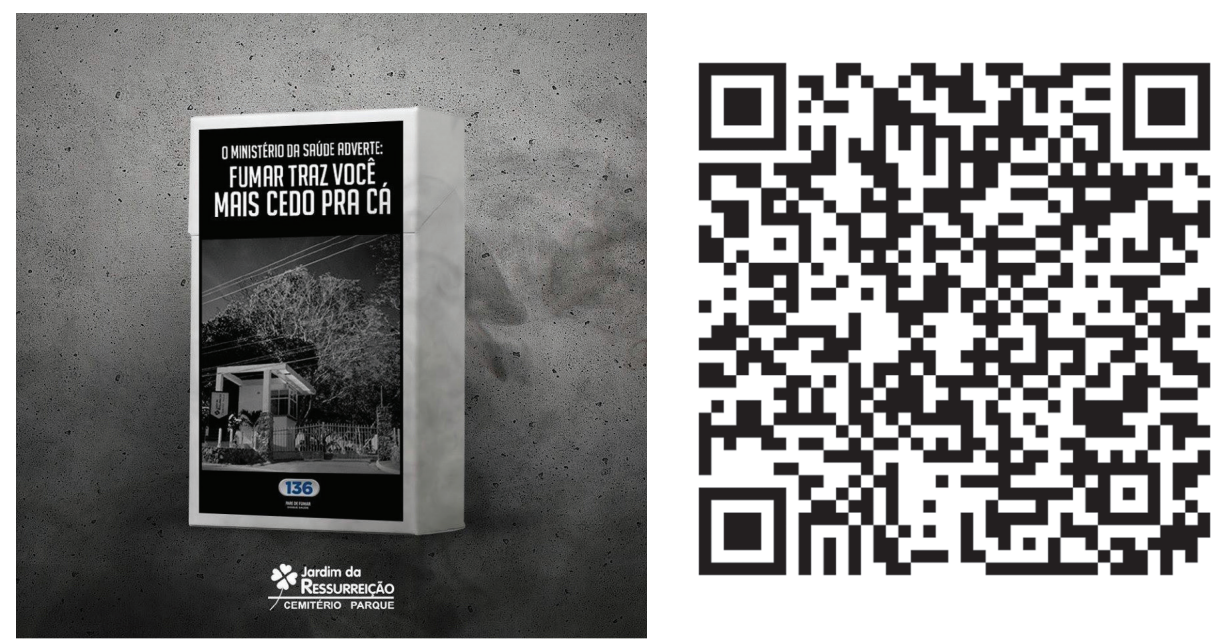

Annonce 2 : Journée Mondiale de Lutte contre le Tabac.

Source: Page Facebook du Cimetière Jardin de la Résurrection

La seconde publication analysée présente le texte suivant : «Le Ministère de la Santé informe : fumer vous fait arriver plus vite ici. 136 - Appelez la Santé. Arrêtez de fumer [logo du cimetière] ». Il est bien clair qu'il s'agit d'une parodie des annonces publicitaires anti-tabagisme du Ministère de la Santé du Gouvernement Fédéral brésilien. La parodie, selon Maingueneau (1997), est une variante du processus d'imitation et se partage entre deux stratégies : la captation et la subversion d'un discours par un autre. Selon ce linguiste, lorsqu'un être parlant s'efface derrière le locuteur d'un genre spécifique de discours et met en évidence ce qu'il fait, il pourra vouloir bénéficier de l'autorité liée à ce type d'énoncé ou la détruire.

Dans le cas de la captation, l'énoncé s'efforce de capter la valeur pragmatique de l'autre à son propre avantage. Par ailleurs, il y a subversion lorsque le texte qui imite cherche à disqualifier, confondre ou désordonner le texte imité, ce qui configure la parodie. C'est ce que l'on peut observer sur le document publicitaire en question qui imite l'esthétique et langage d'une campagne publicitaire anti-tabagisme du gouvernement mais qui la détourne dans un but humoristique afin de surprendre le lecteur par la coupure de la continuité du discours.

Le procédé de parodie introduit un effet de bivocalité dans le texte qui imite (Maingueneau 1997) : la voix de l'imité et la voix qui imite sont présentes et diluées l'une dans l'autre. L'autre sera tout d'abord imité et ensuite détourné. Pour imiter et détourner l'autre, la parodie viabilise des ressources pour la reconnaissance des thèmes et des figures enchaînées et traitées de manière propre dans le discours de référence. Et c'est justement la parodie élaborée qui 
fixe la présence de l'humour et implique l'internaute : en premier lieu, par la reconnaissance et la sensation de familiarité avec la construction visuelle et verbale présentée dans la composition de l'annonce et, par la suite, par le biais de la surprise provoquée par le discours subversif dans l'élaboration créative du message.

L'effet d'humour est renforcé par l'image mise au dos des paquets de cigarettes. Les publicités traditionnelles anti-tabagisme diffusées par le Ministère de la Santé présentent, au même endroit, des images de personnes sévèrement fragilisées par des maladies liées à la consommation de tabac. Cependant, dans la publication élaborée par Cemi, c'est l'image de l'entrée du cimetière qui a été postée, ce qui renvoie à l'idée de l'ultime conséquence de la consommation de tabac : l'enterrement. Le ton plus dramatique et autopromotionnel de la publication est également un élément d'affirmation de l'humour.

Rappelons-nous que l'humour a déjà été étudié par Barreto en 1982 en tant que stratégie discursive de la publicité lorsqu'il travaillait sur la créativité éditoriale. Selon cet auteur, l'humour fait vendre. Il pondère néanmoins ce propos et attire l'attention sur le soin à apporter en utilisant des jeux de mots qui peuvent très souvent paraître « infâmes ".

La présence de l'humour dans les stratégies de communication organisationnelle et de création publicitaire du Cimetière Jardin de la Résurrection configure ce que Sant'Anna et al. (2013) appelle approach : " [c'est la meilleure approche, de séduction, pour persuader le consommateur. Son choix est extrêmement stratégique et facteur de succès ou d'échec de toute la campagne » (Sant'Anna et al., $2013 ; 180-181$ ).

Hormis le retour commercial, l'humour a également le potentiel de rendre visible des inadéquations et problèmes et peut avoir des tactiques de résistance pour "déstabiliser, opposer et juxtaposer les sens de façon à créer un sentiment d'étrangeté et à fixer de nouveaux sens " (Marques \& Oliveira 2012 :106). Nous pouvons ainsi problématiser, dans le cas de cette seconde publication, les propres tactiques organisationnelles utilisées dans les campagnes anti-tabagisme gouvernementales puisqu'il s'agit d'une critique à un mécanisme non innovant et dont les stratégies ont été incorporées par le sens commun et naturalisées, ce qui diminue ainsi la force de pression sur l'industrie du tabac. Il est également juste de penser que, si l'on tient compte du processus de médiatisation, il existe des sens qui circulent et créent des points de fuite, des appropriations imprévues, comme celle de la logique des campagnes anti-tabac, inattendue peut-être de la part d'une organisation qui, ironiquement, aurait intérêt à ce que l'industrie du tabac augmente sa clientèle (bien qu'indirectement). 

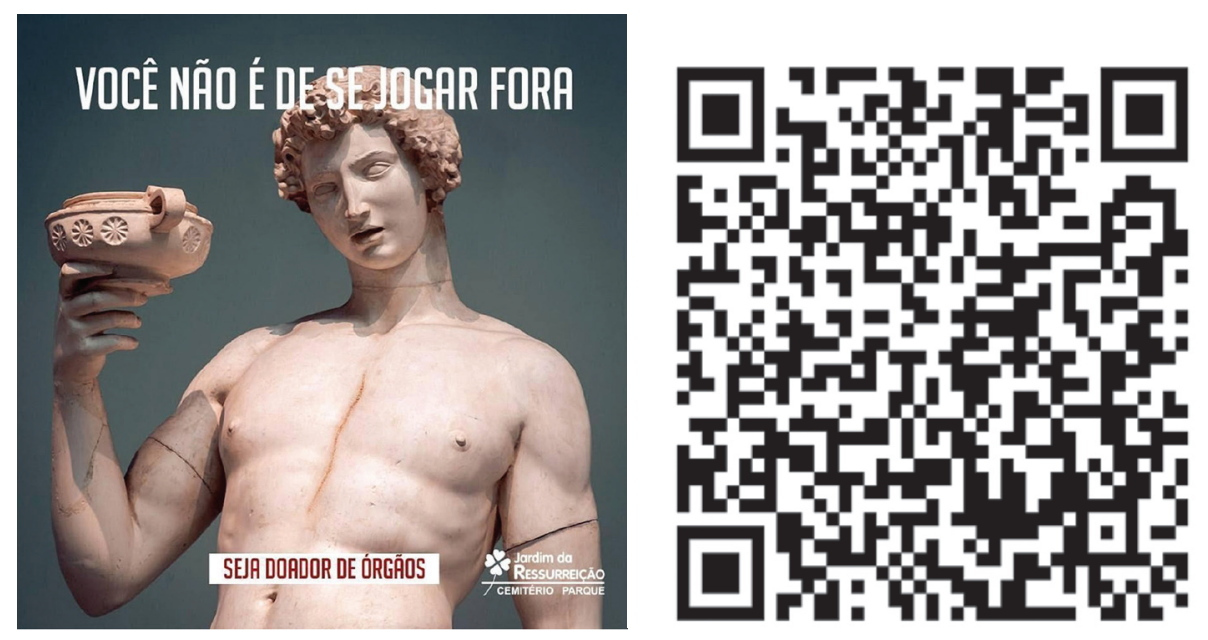

Annonce 3 : Campagne pour les Dons d'Organes

Source : Page Facebook du Jardin de la Résurrection

La troisième publication contient l'énoncé suivant : «Tu n'es pas fait pour être jeté à la poubelle. Sois donneur d'organes [logo du cimetière] ». L'énoncé contient une " manœuvre de séduction » bien connue dans l'imaginaire collectif des internautes. Il s'agit d'un compliment consolidé par le refus de la locution dépréciative. Ce jeu d'oppositions rapproche le discours de l'humour et engendre une incitation émotionnelle auprès des lecteurs, comme selon Berger (2014 : 112-113) :

Bien qu'il soit tentant de dire que ces choses soient devenues virales juste parce quelles sont amusantes, un processus plus fondamental a lieu. [...] Comme les choses qui nous inspirent ou nous rendent furieux, le contenu humoristique est partagé car le divertissement est une émotion de grande excitation.

L'humour est renforcé par le potentiel polysémique (Brait 2005), ou au double sens, attribué à l'expression « jeté à la poubelle ». Le sens connotatif - plus connu et utilisé populairement - fait allusion au sentiment de désintérêt, à l'idée de ne pas prendre en considération une personne car elle n'est pas assez bonne ou attirante, ou encore car elle ne dispose pas de qualités ou d'attributs suffisamment intéressants. Le sens dénotatif, quant à lui, renvoie à l'idée réelle de rejet, d'inutilisation, de perte et d'anéantissement liée à la mort. Et c'est justement ce sens qui fait le lien avec la campagne de dons d'organes. Ce lien est corroboré par l'image de la statue qui présente des marques nettes et visibles de séparation des membres qui s'apparentent à des cicatrices chirurgicales. La statue utilisée dans la pièce graphique du Cemi est en lien avec l'imaginaire construit autour de l'espace des cimetières. En 
effet, les cimetières les plus anciens étaient envahis de statues qui ornaient les tombes et les mausolées.

Le discours de cette publication présente un énoncé qui, à première vue, semble être le mème ${ }^{7}$ d'un « flirt bon marché » mais qui, à la fin, se révèle une préoccupation sociale et noble dont l'intention est d'éviter que les organes soient littéralement " jetés à la poubelle » alors qu'ils pourraient continuer à fonctionner pour sauver d'autres vies.

L'appropriation des mèmes, comme celle des jeux de mots et expressions utilisées fréquemment par le public de jeunes sur les réseaux sociaux, facilite la mobilisation et la circulation du contenu de cette page du Facebook, diffuse et humanise l'organisation malgré les difficultés associées à la construction de la marque d'un cimetière. Bien que des innovations aient été identifiées sur la page, l'on peut noter que de nombreuses stratégies sont appropriées à partir de cas qui ont fait succès, comme le cas de la Mairie de Curitiba que nous pouvons citer ici (Coelho 2015) qui fait également recours à l'humour, à des narrations trans-médias et au lien avec des thématiques d'intérêt public en allant au-delà des obstacles de communications et des stigmatisations associées à certains segments organisationnels.
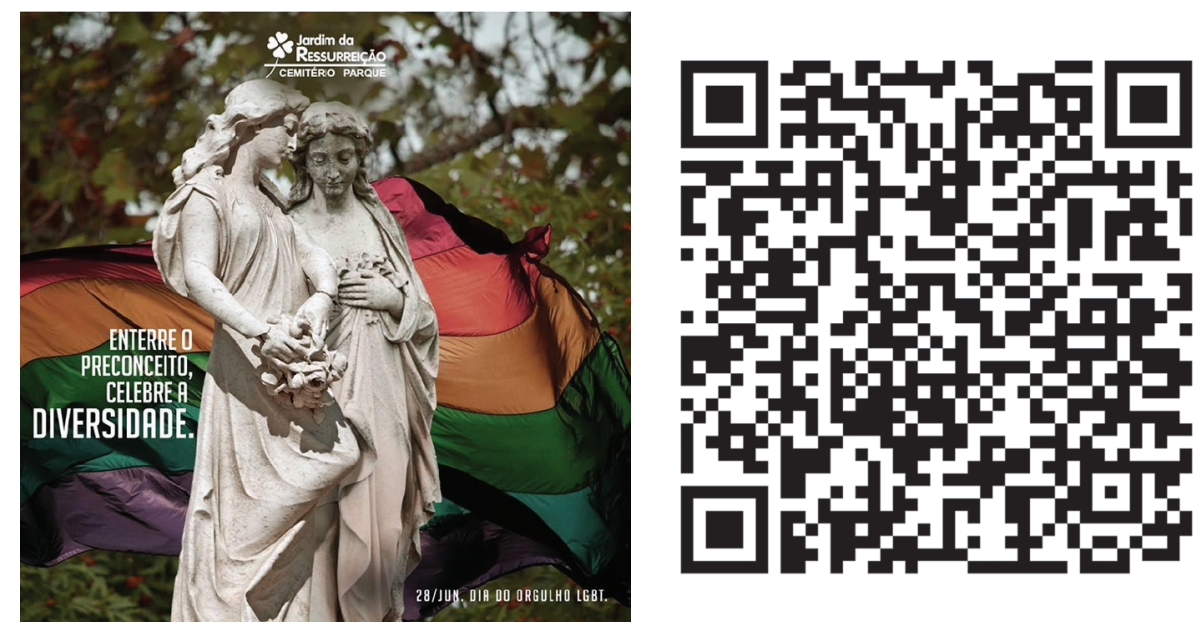

Publication 4 - Gay Pride

Source : Page Facebook Cimetière Jardin de la Résurrection

La dernière publication analysée présente l'énoncé suivant : " [logo du cimetière] Enterre les préjugés et célèbre la diversité. 28 juin. Gay Pride LGBT ». Le point fort de l'énoncé est centré sur le verbe enterrer à l'impératif (enterre) qui permet d'explorer la polysémie (Brait 2005) ou de

7 L’expression mème est utilisée pour décrire le concept du magnet comique. 
diverses significations associées au même mot. Le sens connotatif renvoie à l'idée de fin, d'extermination et de destruction. Le sens dénotatif, toutefois, fait référence à l'idée de sépulture, d'enterrement, d'enfouissement et établit une connexion plus directe avec l'organisation. Lacte "d'enterrer » est la prestation principale d'un cimetière et, c'est pour cette raison que l'énoncé est tellement approprié, opportun et créatif. En outre, la mort des préjugés serait certainement massivement soutenue par les internautes.

Il existe aussi dans l'énoncé une critique puissante et implicite dans l'énoncé de cette publication, toujours en lien avec le verbe « enterrer », pour dénoncer les nombreux cas de phobie contre la communauté LGBT qui ont fait des victimes mortelles au Brésil. Si le verbe évoque la mort, la relation entre la mort et la population LGBT, facile à établir dans notre contexte contemporain, est subversive pour confondre une critique sociale qui tente d'éliminer et d'enterrer (mettre un terme définitivement) le préjugé pour valoriser la vie de cette partie de la population.

Cette publication analysée, hormis la promotion de l'organisation qui en est l'auteur, a également comme objectif de susciter l'engagement envers la cause sociale diffusée. Toute l'atmosphère symbolique élaborée dans sa composition visuelle renforce cette intention. Au fond, on peut voir le drapeau de la Gay Pride qui flotte. Devant le drapeau, se trouvent deux statues aux formes féminines. Il est intéressant de noter que l'humour, qui est souvent utilisé pour disqualifier la communauté LGBT (Irigaray et al.2010) est approprié ici dans le sens inverse des valeurs hétéro-normatives.

Il est juste d'observer que l'humour a été employé de forme discrète afin de créer un climat de décontraction sans compromettre le sérieux de la cause sociale défendue. Deligne (2011) souligne qu'il est nécessaire de prendre des précautions lorsque l'on fait recours à l'humour dans les stratégies de communication car, tout compte fait, il peut y avoir des problèmes d'interprétation dans le message et l'auteur peut ne pas être compris. À cet effet, il fait usage du « principe de coopération » (Deligne 2011 : 40) selon lequel l'auteur du message humoristique doit respecter quatre règles pour être compris : 1) la quantité (donner le nombre d'informations nécessaires et pas plus) ; 2) la qualité (ne pas affirmer ce qui est faux) ; 3) la relation (être bien clair dans ce que l'on veut dire) et 4) la modalité (éviter l'ambiguité).

Le principe de la coopération est également lié, dans le cas de la quatrième publication et de bien d'autres diffusées par Cemi, à la reconnaissance de problèmes collectifs (Marques \& Oliveira 2012), comme la phobie des LGBT, la lutte contre le tabagisme et le suicide ainsi que la nécessité d'augmenter les dons d'organes. Cette reconnaissance est également associée à un projet politique d'une société fondée sur la tolérance, qui minimise les objectifs commerciaux face à la perspective de l'engagement et qui est déjà employé 
au sein des stratégies d'autres entreprises, comme Benetton (d'Almeida 2007). Ces questions touchent le public du cimetière sur Facebook car elles l'engagent individuellement et collectivement.

\section{Considérations finales}

À partir des analyses développées, on peut observer que l'approche de la page Facebook du Cimetière du Jardin de la Résurrection introduit l'humour en lien à la sensibilité lorsqu'il s'agit de traiter de questions délicates et renvoie à la mort de façon amusante mais avec une extrême prudence et créativité. La mort, thème encore tabou au Brésil et dans l'état du Piauí et qui entraîne une grande résistance, se voit déconstruite dans le cadre d'un processus de promotion du cimetière. Elle est néanmoins utilisée pour mobiliser le public autour d'importantes campagnes de valorisation de la vie en stimulant d'importants débats, comme celui de la prévention du suicide, qui sont du ressort de l'intérêt public.

Nous avons pu identifier certaines stratégies et tactiques employées dans le discours des annonces sélectionnées. Nous avons noté que le recours à l'humour est basé sur des mécanismes bien articulés, comme c'est le cas du lien avec : 1) la «monnaie sociale " et le « capital social » afin d'octroyer une valeur aux communications publicitaires pour véhiculer des éléments qui appartiennent à l'univers symbolique des internautes ; 2) l'élaboration de parodies qui stimulent une reconnaissance aisée et l'identification du public et qui permettent une coupure de continuité en stimulant son attirance ; 3) l'incitation à l'excitation émotionnelle du public et 4) l'utilisation de recours linguistiques, comme la polysémie, qui permet un jeu à multiples sens et un facteur de surprise pour le public.

Il est notoire que l'humour est aussi un recours stratégique déterminant pour renforcer et potentialiser l'image du Cimetière du Jardin de la Résurrection. Son utilisation est faite sur la base de l'articulation d'éléments-clés qui garantissent le sérieux de l'approche des causes sociales tout en offrant une atmosphère discursive qui est soulignée par la légèreté, même lorsqu'il existe des éléments subversifs implicites dans les annonces pour questionner les sens dominants. Les stratégies organisationnelles sont articulées à une inventivité discursive sur Facebook bien qu'elles fassent recours à des symboles et des sens largement diffusés sur internet.

Cette articulation fut principalement essentielle pour la communication et la diffusion du cimetière de manière attirante et captivante tout en tenant compte qu'il est porteur d'une thématique qui fait l'objet d'aversion et d'antipathie (la mort). Le recours à l'humour en tant que tactique discursive est configuré comme une stratégie efficace de communication dans le cas du Cemi. On peut vérifier les indices d'une communication organisationnelle de succès de par le nombre de j'aime, de réactions, de partages et de commentaires au sujet des annonces qui, bien qu'elles ne soient pas exploitées de façon 
profonde dans notre analyse, constituent un retour important pour l'équipe qui gère la page Facebook car elles indiquent les actions qui ont conquis ou non le public.

\section{BIBLIOGRAPHIE}

BARRETO Roberto Menna, 1982, Criatividade em propaganda, São Paulo, Summus. BERGER Jonah, 2014, Contágio: por que as coisas pegam, Rio de Janeiro, LeYa.

BERGSON Henri, 1983, O riso: ensaio sobre o significado do cômico, Rio de Janeiro, Zahar.

BRAIT Beth (coord.), 2005, Bakbtin: conceitos-chave, São Paulo, Contexto.

CARRIERI Alexandre de Pádua, 2004, "O humor como estratégia discursiva de resistência: as charges do SINTTEL/MG ", Organizaçôes E Sociedade, vol. 11, no 30, p. 29-48.

COÊLHO Tamires Ferreira, 2015, " Midiatização e Estratégias da Prefeitura de Curitiba no Facebook durante a Campanha \#CasamentoVermelho », in MARQUES Angela \& OLIVEIRA Ivone (coord.), Comunicação organizacional: dimensöes epistemológicas e discursivas, Belo Horizonte, FAFICH/UFMG, vol. 1, p. 54-69.

FAUSTO NETO Antonio, «A circulação além das bordas », In FAUSTO NETO Antonio \& VALDETTARO Sandra (coord.), Mediatización, sociedad y sentido, vol. 1, p. 2-17.

D'ALMEIDA Nicole, 2007, Les promesses de la communication, Paris, PUF.

D'ALMEIDA Nicole, 2015, «O estatuto do sujeito em organização », in MARQUES Angela \& OLIVEIRA Ivone (coord.), Comunicação organizacional: dimensöes epistemológicas e discursivas, Belo Horizonte, FAFICH/UFMG, vol. 1, p. 18-26.

DELIGNE Alain, 2011, « De que forma o riso pode ser considerado subversivo ? » in LUSTOSA, Isabel (coord.), Imprensa, bumor e caricatura: a questão dos estereótipos, Belo Horizonte, UFMG.

GOMES Juliana et al., 2016, "Mídia Social como ferramenta estratégica de Comunicação Organizacional: um estudo de caso sobre o Cemitério Jardim da Ressurreição ", in XXXIX Congresso Brasileiro de Ciências da Comunicação, São Paulo. Disponible sur: <http://portalintercom.org.br/anais/nacional2016/resumos/R11-11411.pdf>.

GUYOT Brigitte, 2012, Management de l'information dans les organisations: éléments de méthode. Disponible sur: <https://archivesic.ccsd.cnrs.fr/sic_00665257/document>.

IRIGARAY Hélio Arthur Reis et al., 2010, « Humor e discriminação por orientação sexual no ambiente organizacional ", RAC - Revista de Administração Contemporânea, vol. 14, no. 5, sep/oct, p. 890-906.

LIQUÈTE Vincent (coord.), 2014, Cultures de l'information. Paris : CNRS éd. (Les essentiels).

MAINGUENEAU Dominique, 1997, Novas tendências em análise do discurso, Campinas, Pontes. 
MARQUES Ângela Salgueiro \& OLIVEIRA Luciana, 2012, « Poder e Resistência: breve reflexão teórica sobre o papel do humor nos conflitos público-privado em contextos organizacionais », Ciberlegenda, no 26, p. 99.

MUMBY Dennis, 2010, "Reflexões críticas sobre comunicação e humanização nas organizações», in Kunsch, Margarida, A comunicação como fator de humanização das organizações, São Caetano do Sul, Difusão, p. 19 -39.

PEREIRA Vinícius Andrade, 2012, "Linguagens midiáticas, entretenimento e multissensorialidade na cultura digital », in REGIS Fátima (coord.), Tecnologias de comunicação e cognição, Porto Alegre, Sulina.

SANT'ANNA Armando et al., 2013, Propaganda: teoria, técnica e prática, São Paulo, Cengage Learning.

SIQUEIRA FILHO Ademir, 2011, "O humor no estudo da humanização da comunicação organizacional ", Revista Comtempo, vol. 3, no 1, jun/nov. Disponible sur: <http://www.revistas.univerciencia.org/index.php/comtempo/article/ viewFile/7562/7214>.

XAVIER Adilson, 2007, O deus da criação, Rio de Janeiro, BestSeller.

Résumé: Notre approche est relative à l'appropriation de l'humour dans la construction des stratégies de communication, plus particulièrement pour traiter un thème difficile (la mort) et à partir d'un modèle d'organisation classiquement peu documenté (un cimetière). Nous avons analysé les tactiques de la page Facebook du cimetière Jardim da Ressurreição (le jardin de la résurrection). Nous avons choisi quatre annonces qui ont été élaborées pour quatre campagnes de diffusion du cimetière et nous réfléchissons sur les stratégies narratives mobilisées dans la construction de l'image institutionnelle du cimetière par le biais du réseau social.

Mots-clés: mort, humour, stratégies de communication organisationnelle, Facebook.

Abstract: We approach the appropriation of humor in the construction of communication
strategies, more specifically the treatment of a challenging theme (death) from an organization
model that is conventionally poorly researched: a cemetery. We analyse tactics on Resurrection
Garden Cemetery (Cemitério Jardim da Ressurreiça) Facebook fanpage from four advertising
campaigns, thinking, more specifically, about the narrative strategies that were mobilized into
the construction of cemetery's institutional image through the social network.

Keywords: death, humor, organizational communication strategies, Facebook. 